\title{
Decode a ticking time-bomb
}

\author{
Song Wan $^{1}$, Jun Liu ${ }^{2}$ \\ ${ }^{1}$ Division of Cardiothoracic Surgery, Department of Surgery, The Chinese University of Hong Kong, Prince of Wales Hospital, Hong Kong, China; \\ ${ }^{2}$ Department of Mechanical Engineering, City University of Hong Kong, Hong Kong, China \\ Correspondence to: Song Wan, MD, FRCS. Division of Cardiothoracic Surgery, Department of Surgery, The Chinese University of Hong Kong, \\ Prince of Wales Hospital, Hong Kong, China. Email: swan@surgery.cuhk.edu.hk. \\ Provenance and Peer Review: This article was commissioned by the editorial office, fournal of Thoracic Disease. The article did not undergo external peer \\ review. \\ Comment on: Wu J, Qiu J, Xie E, et al. Predicting in-hospital rupture of type A aortic dissection using Random Forest. J Thorac Dis 2019;11:4634-46.
}

Submitted Apr 26, 2020. Accepted for publication May 09, 2020.

doi: 10.21037/jtd-2020-52

View this article at: http://dx.doi.org/10.21037/jtd-2020-52

Acute type A aortic dissection is arguably the number one life-threatening cardiovascular surgical emergency, and its incidence is increasing in China. The numbers of surgical operations performed for diseases of the thoracic aorta in China were 15,593 in 2016, 19,585 in 2017, and 22,898 in 2018 (1). It is estimated that at least half to approximately two thirds of these operations were related to aortic dissection (including both Stanford types A and B), although the exact number of operations for type A dissection was not reported for each year. Type A aortic dissection is usually considered as a "ticking time-bomb." Without prompt surgical intervention, a mortality rate of $1 \%$ per hour over the first 48 hours is commonly cited. Such a "clear and present danger" indeed represents a serious challenge that faces the entire community of cardiovascular patients nationwide.

At the institutional level, one of the most reliable track records in China has been achieved by the Fuwai Hospital of Chinese Academy of Medical Sciences in Beijing. As the National Center for Cardiovascular Disease, Fuwai Hospital is the largest specialized flagship institution in China with an impressive annual cardiac surgery volume exceeding 14,000 (e.g., 14,899 in 2017 and 14,455 in 2018) (1). For instance, 520 of the 1,474 aortic procedures conducted at this institution in 2018 were performed for aortic dissection (including 272 emergency procedures for type A aortic dissection) (2). Momentously, based on their institutional data involving 1133 consecutive patients with type A aortic dissection over a recent 7-year period, a random forest classification model for prediction of in-hospital aortic rupture was developed and internally validated by $\mathrm{Wu}$ and colleagues from Fuwai Hospital (3). With the aim of scientifically risk stratifying this dangerous disease and prioritizing the timing of operation according to each patient's pathological characteristics, they meticulously selected and evaluated 16 important clinical variables (3). Consequently, a relevant and timely tool was developed that might substantially impact the decision-making process for aortic surgeons-particularly for young resident surgeons who are still in training.

A logical next step would be to externally validate this model using prospective multicenter data from an even larger sample size. However, it is noteworthy that the Fuwai Hospital dataset, as $\mathrm{Wu}$ and colleagues presented, is somewhat unique in terms of the very young age group of patients (mean age, 49.5 years) and the relatively high incidence of preoperative in-hospital aortic rupture (116 of the 1,133 patients). We suspect that this was at least partially influenced by the nature of the hospital. As the largest referral center for cardiovascular diseases in China, Fuwai Hospital receives many patients from nearby cities and even other provinces. In this studied patient cohort, $\mathrm{Wu}$ and colleagues did not report the detailed durations from the onset of symptoms to the establishment of type A aortic dissection diagnosis or to the time of operation. It remains unclear whether such potentially "prolonged" durations contributed to the $10 \%$ incidence of preoperative aortic rupture. Meanwhile, the exact mortality rate in the 116 patients who had preoperative aortic rupture was also unknown (although we understand that the risk of death 

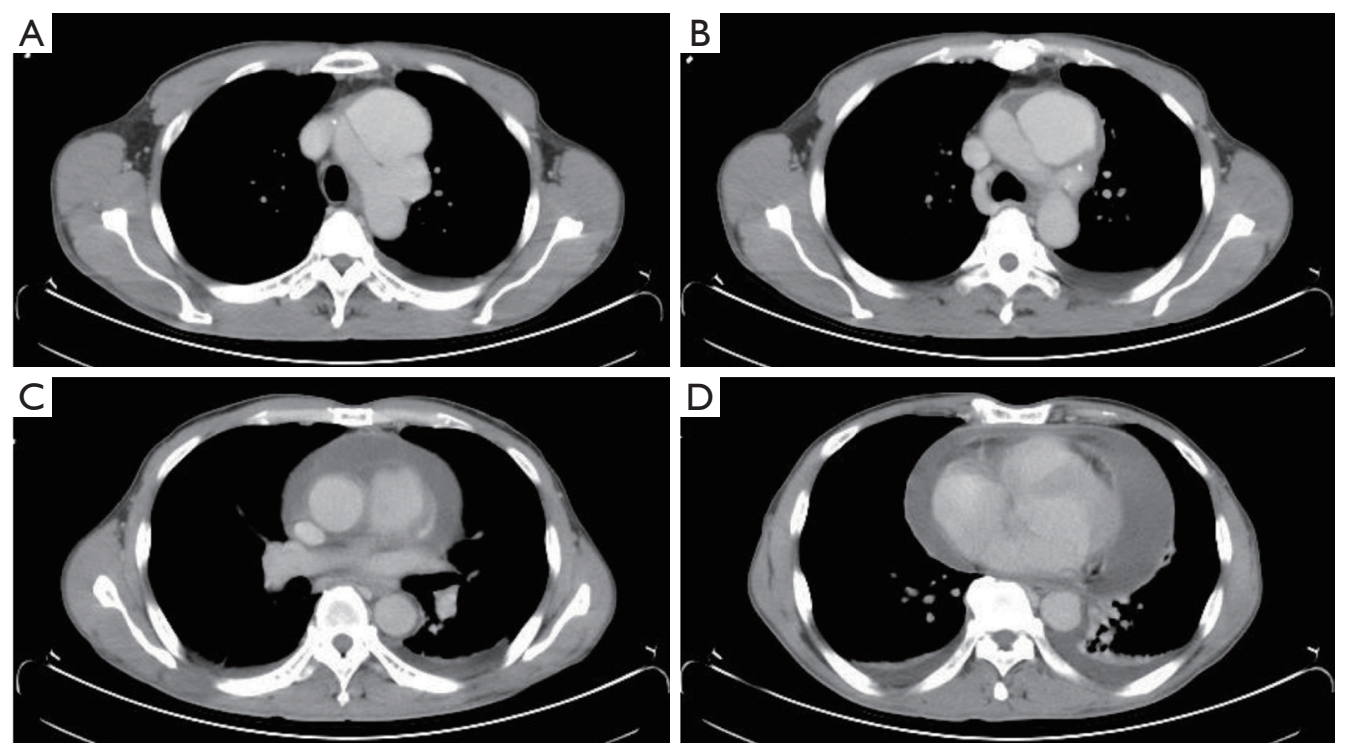

Figure 1 Representative computed tomographic images from a patient with a contained aortic arch dissecting aneurysm and significant amount of pericardial effusion.

could be extremely high in this group of patients).

From the clinical points of view, several additional considerations may deserve further discussion. On the one hand, are the current 16 "important variables" selected by $\mathrm{Wu}$ and colleagues good enough to predict aortic rupture? Although a positive answer is indeed very likely, there may still be rare exceptions. As an example, I (S.W.) can recall a patient on whom I operated a few years ago; the dissecting aorta ruptured in front of my eyes:

The patient was a 55-year-old man with good past health who was transferred to our hospital on a late evening (around 22:30 pm) following his "incidental finding" of type $A$ aortic dissection (Figure 1). He had chest pain radiating to the back for 2 days and was undergoing elective clinical investigations at a nearby private hospital to rule out "possible malignancy." The results of his series of examinations, including blood tests, echocardiography, abdominal ultrasound, esophagogastroduodenoscopy, and colonoscopy, were all normal, until the PET-CT scan revealed aortic arch dissection. Interestingly, both the entry and the exit tears were within the aortic arch (Figure 1A,B), which resulted in a buge contained dissecting aneurysm (measuring $7.1 \mathrm{~cm} \times 3.7 \mathrm{~cm} \times 6.7 \mathrm{~cm}$ ). However, the ascending and descending parts of the aorta were largely spared from dissection (Figure 1C). Substantial pericardial effusion was also detected (Figure 1C,D). An urgent operation commenced at 2:00 am. Surprisingly, the pericardial fluid appeared clear and was not blood-stained at all (i.e., it was not a hemopericardium in the first place!). Nonetheless, as expected, the dissecting arch aneurysm ruptured as soon as the cardiac tamponade was relieved (and the blood pressure shot up). Cardiopulmonary bypass via pre-prepared femoral cannulation was quickly established, and while the rupture site was temporarily hand-sealed by my assistant, whole-body cooling was begun immediately. A total arch replacement, with the insertion of a frozen elephant trunk, was then performed. The patient made an uneventful recovery, was extubated 6 hours after the procedure, transferred out of the intensive care unit the next day, and discharged home a week later.

Obviously, this was not a typical case of acute type A aortic dissection. The actual onset time of his dissection remained uncertain, the four most important variables proposed by $\mathrm{Wu}$ and colleagues were absent (periaortic hematoma, hemopericardium, lower-limb numbness/pain, and syncope). The decision to perform an urgent lifesaving operation without any delay was purely based on the surgeon's "gut feeling" of a high risk of aortic rupture. Thus, few would disagree that even if all existing risk score systems have been proven to be extremely valuable, they are meant to be complementary and can never completely replace a physicians' clinical judgement.

On the other hand, can this random forest classification model help us safely delay an operation for several hours and still ensure improved survival for some selected patients with type A aortic dissection? Although this aspect was not fully explored by $\mathrm{Wu}$ and colleagues, a positive answer is again very likely. In many hospitals worldwide, the night-shift 


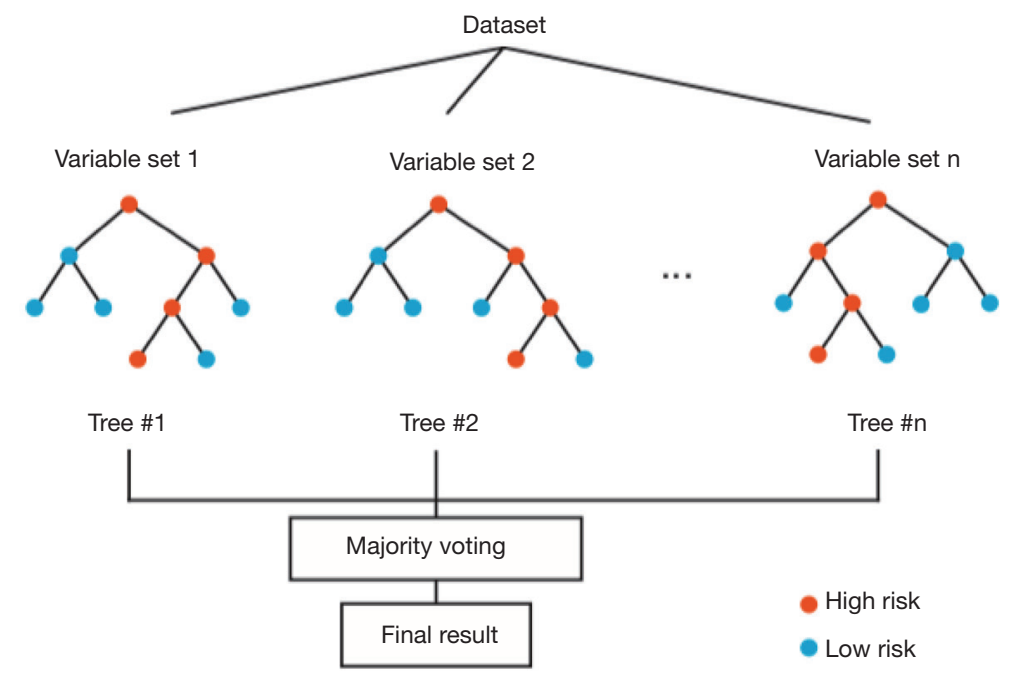

Figure 2 Conceptual diagram of random forest algorithm. Averaging of many random decision trees significantly reduces the variance of and bias in individual samples.

on-call surgeons are often "on-call 24 hours". This means that they have to perform their grueling routine clinical duties during the day before commencing the evening tasks. Moreover, the least manpower is usually available during the night shift. For the above-mentioned case, the complex operation was performed by a surgeon and a resident doctor in training. In such a scenario, in case any urgent assistance is required, it would be difficult to get another helping hand. Therefore, instead of pushing an already tired surgical team to "run extra miles" (i.e., performing a mentally challenging and physically demanding emergency aortic operation starting at midnight or in the very early morning), having a "fresh and energetic" team perform the procedure at 8:00 am carries obvious advantages. Such an arrangement could ultimately create safer conditions for patients. Hence, as long as proper monitoring and medical therapy have been adequately provided in this particular setting, delaying a surgical intervention for a few hours in some carefully evaluated patients might not be a bad thing. A recent study (4) reported this to be a well-accepted practice, wherein the majority of operations (up to 80-90\%) for acute type A aortic dissection were actually performed during the day shift, and many patients had waited a median duration of 10-15 hours before undergoing the operation. In this regard, $\mathrm{Wu}$ and colleagues' predictive model could be a strategic tool to ensure good clinical outcomes.

Notably, Wu and colleagues demonstrated that the random forest is one of the best choices for classification and prediction. The random forest fundamentally is a forest of decision trees combined to analyze the categorical features (5). In the training stage, the random forest model repeatedly applies the bootstrapping or bagging technique to select a random sample and fits in the decision trees that are built with a random subset of feature variables. Although a single decision tree is sensitive to the noise in a single training step, averaging many random trees can reduce the variance of the model and minimize the bias in individual samples and feature variables (Figure 2). Examination of the out-of-bag error in the random forest model can also rank the importance of the feature variables. Compared with other classification algorithms, the random forest algorithm has a high prediction accuracy and can process highdimensional data and a large number of training samples. Because multiple factors could be the indicators to predict rupture after type A aortic dissection, the random forest model is a suitable method for analysis of the complicated factors and rank their importance for the assessment of rupture risk. Because the random forest model is built upon a group of decision trees, it is relatively easy to implement and has a low computational cost. Moreover, as the sample size and the feature space increase, the averaging of the decision trees in the random forest model can reduce sample variance and the tendency of overfitting, thus making large ensembles more feasible. Although it is easy to build and train a random forest model, the clinical use of this model may have practical issues of shifting the computation from training time to evaluation time (6). In addition, a relatively high number of variables are collected 
from a single patient to feed in this model, which might be very time-consuming for the assessment of rupture risk in type A aortic dissection. As shown by $\mathrm{Wu}$ and colleagues, the random forest model requires more variables than the Lasso regression model, and the validation confirmed that the random forest and Lasso models share a significant overlapping of the most critical factors, including periaortic hematoma, hemopericardium, and lower-limb numbness/ pain.

In summary, $\mathrm{Wu}$ and colleagues reported the first attempt to quantitively evaluate the risk of in-hospital rupture for patients with type A aortic dissection using a machine learning method. This model based on the random forest algorithm is a useful tool for predicting rupture with a relatively large number of variables. The analysis of high-dimensional data and mining of their underlining correlations with rupture could assist surgeons during the initial screening of an acute case and relieve them from the tedious examination of many variables. It is granted that clinical decisions should be individualized and that the timing of surgery should always be determined according to each patient's specific conditions. In addition to the clinical value of evaluating the rupture risk in aortic dissection, $\mathrm{Wu}$ and colleagues' work also presents a pioneering example for the analysis of large-scale data by applying a machine learning method. In the foreseeable future, this study may inspire more researchers to investigate new intelligent algorithms for the risk stratification and management of thoracic diseases.

\section{Acknowledgments}

Funding: None.

\section{Footnote}

Conflicts of Interest: Both authors have completed the ICMJE uniform disclosure form (available at http://dx.doi. org/10.21037/jtd-2020-52). The authors have no conflicts of interest to declare.

Ethical Statement: The authors are accountable for all aspects of the work in ensuring that questions related to the accuracy or integrity of any part of the work are appropriately investigated and resolved.

Open Access Statement: This is an Open Access article distributed in accordance with the Creative Commons Attribution-NonCommercial-NoDerivs 4.0 International License (CC BY-NC-ND 4.0), which permits the noncommercial replication and distribution of the article with the strict proviso that no changes or edits are made and the original work is properly cited (including links to both the formal publication through the relevant DOI and the license). See: https://creativecommons.org/licenses/by-nc-nd/4.0/.

\section{References}

1. Chinese Society of Extracorporeal Circulation. White book of Chinese cardiovascular surgery and extracorporeal circulation in 2017 and 2018. Chin J ECC 2019;17:257-60.

2. Cardiovascular surgery outcomes 2018. Available online: http://www.fuwai.com/News/Articles/Index/211815

3. Wu J, Qiu J, Xie E, et al. Predicting in-hospital rupture of type A aortic dissection using Random Forest. J Thorac Dis 2019;11:4634-46.

4. Umana-Pizano JB, Nissen AP, Sandhu HK, et al. Acute type-A dissection repair by high versus low volume surgeons at a high-volume aortic center. Ann Thorac Surg 2019;108:1330-6.

5. Breiman L. Random Forests. Mach Learn 2001;45:5-32.

6. Gashler M. Decision tree ensemble: small heterogeneous is better than large homogeneous. Seventh International Conference on Machine Learning and Applications (ICMLA) 2008:900-905.
Cite this article as: Wan S, Liu J. Decode a ticking timebomb. J Thorac Dis 2020;12(9):4598-4601. doi: 10.21037/jtd2020-52 